\title{
Book Review \\ Diskina, D.E., Shabalina, T.N., Zanozina, I.I., and Tyshchenko, V.A., Khromatograficheskie i Termoanaliticheskie Issledovaniya Masel $i$ Rabochikh Zhidkostei (Chromatographic and Thermoanalytical Studies of Oils and Working Fluids),
} Samara: Ofort Open Company, 2011, 160 p.

DOI: $10.1134 / \mathrm{S} 1061934812060111$

The authors of this monograph are researchers with a long experience of research into the development of new oils and working fluids, new chromatographic procedures and methods, in some cases using combinations of chromatography with thermal analysis.

The book was published in the International Year of Chemistry and dedicated to outstanding scientists who passed away, Doctor of Technical Sciences Knara Mambreevna Badyshtova, a well-known expert in industrial oils, and Doctor of Chemistry, Professor Mark Solomonovich Vigdergauz, who had done so much for the development and popularization of chromatography in the studies of oils and oil products. The monograph summarized materials on enhancing the methodology of investigating basic and doped industrial oils and special petroleum and synthetic working fluids. Three main lines of research can be distinguished: the development of rapid tests to analytically control the manufacture of the above products; the development of rapid methods for estimating their "specialized" working characteristics by gas chromatography, including its reaction versions; attraction of thermal analysis previously used only in inorganic and polymer analysis.
Chapter 1 of the monograph presents a description of the theoretical basics of the main methods developed by the authors. The next three chapters include a detailed description of the developed and improved methods and procedures introduced at a number of Russian petroleum plants (Kstovo, Ufa, Kirishi, Novokuibyshevsk, Syzran, Perm, Angarsk, etc.) and at different enterprises (Research Institute of Nuclear Reactors, etc.). Examples of complex studies of products of unknown composition, use of chromatographic and thermoanalytical methods in the development of oils for biological preparations (anti-FMD vaccine), oils for rocket and missile control systems, for estimating the heat capacity of the ditolylmethane heat-transfer medium of nuclear heating plants, and so on.

The monograph is interesting and useful for workers at research institutions and factory laboratories of the oil and petrochemical industry, as well as for teachers, graduate students, and students of higher educational institutes.

\section{A. Platonov}

